\title{
Synthesis of phenylimidazo thiazolo benzocycloheptene derivatives as potential antiinflammatory agent-IV ${ }^{\dagger}$
}

\section{Lingaiah Nagarapu* and $\mathrm{N}$ Venkateswara Rao}

Division of Urganıc Chemistry-II, Indian Institute of Chemical Technology, Hyderabad-500 007, India.

Abstract : New heterocyclic systems namely 10-phenyl-6,7-dihydro-5Hbenzo[6,7] cyclohepta[1,2-d][1,3]thieazoles $4 a-c$ have been synthesized via the reaction of 2-(2-imino-1,4,5,6-tetrahydro-2H-benzo[7,8]cyclohepta[d][1,3] thiazol)1-phenyl-1-propen-1-ol (3a-c) intermediates with phenacyl bromide, in good yields. All these compounds (4a-c) exhibit significant antiinflammatory activity.

\section{Introduction}

A number of biologically interesting polynuclear compounds incorporating a fused thiophene ring viz. Thiasteroids, ${ }_{1}$ analogues of indole alkaloids, ${ }^{2.3}$ carcinogenic compounds ${ }^{4}$ etc., consists of six-membered ring annelated to thiophene. But examples of polycondensed systems incorporating a thiophene, imidazole or thiazole ring fused to a seven membered ring (viz benosuberones and benzazepines) are sparse. In continuation of our previous studies ${ }^{5-7}$ in the synthesis of biologically active fused heterocycles we have synthesized the hitherto unreported phenylimidazo thiazolobenzocycloheptene derivaties $4 a-c$ starting from the 6,7,8,9-tetrahydro-5H-benzocycloheptene-5-ones $1 \mathrm{a}-\mathrm{c}^{8}$ and their analgesic and antiinflammatory activities studied in the present investigation.

\section{Chemistry}

Reaction of the tetrahydro benzocycloheptenones (1a-c) with thiourea and iodine were heated under reflux to give expected 5,6-dihydro-4Hbenzo[3,4]cyclohepta[d][1,3] thiazol-2-amines (2a-c) as colourless needles (60$65 \%)^{7,9}$ Cyclization of (2a-c) with phenacyl bromide at room temperatur resulted in 2-(2-imino-1,4,5,6 tetrahydro2H-benzo[7,8]cyclohepta [d]][1,3] thiazol-1-yl)-1phenyl-1-propen-ols (3a-c) as the intermediiate products $(67-68 \%)$. Subsequently compounds (3a-c) were assigned the enol form and gave 10-phenyl-6,7-dihydro$5 \mathrm{H}$-benzo[6,7]cyclohepta[1,2-d] imidazo $[2,1-d][1,3]$ thiazoles (4a-c, 90-92\%)

\footnotetext{
${ }^{\dagger}$ IICT Communication No. 4623
} 
heating in ethanol (Scheme-1). Their structures were established by ${ }^{1} \mathrm{H}$ NMR, IR and elemental analysis. These compounds (4a-c) were tested for their analgesic and antiinflammatory activities.

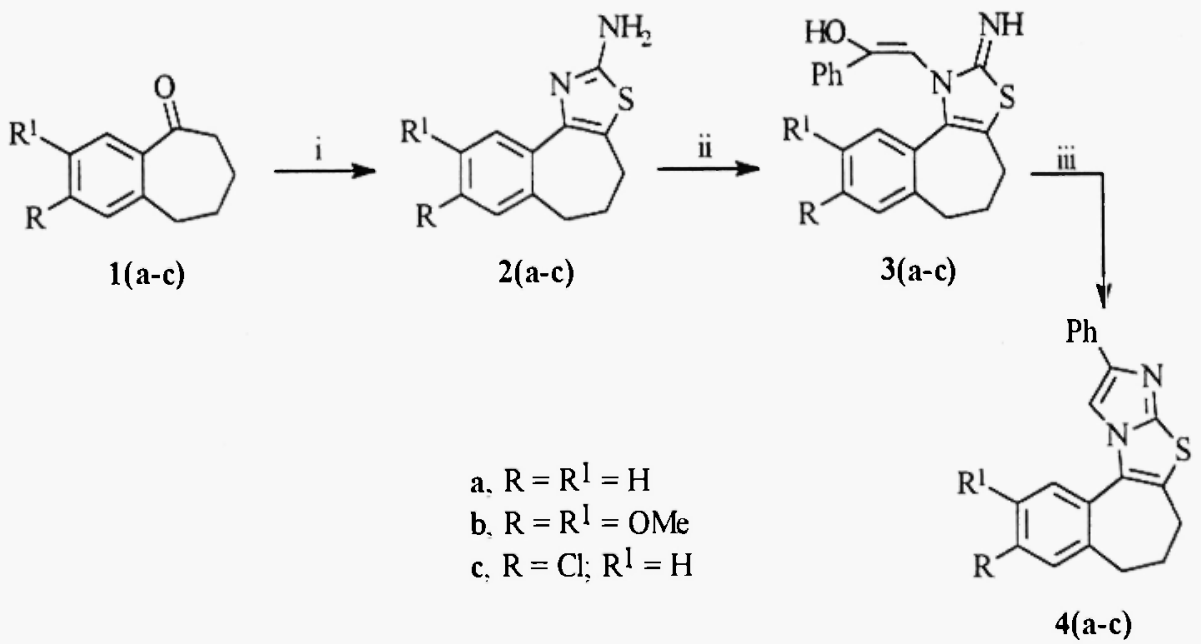

Reagents and Conditions. i) $\mathrm{H}_{2} \mathrm{NCSNH}_{2}, \mathrm{I}_{2}, \mathrm{EtOH}$, reflux; ii) $\mathrm{PhCOCH}_{2} \mathrm{Br}, \mathrm{EtOH}$, $\mathrm{RT}$, overnight; iii) EtOH, reflux, 6 hrs.

\section{Scheme-1}

\section{Analgesic and antiinflammatory activities}

Analgesic and antiinflammatory activities of the compounds $4 a-c$ were determined by Turner ${ }^{10}$ writhing test ${ }^{11}$ and rat-paw edema test. ${ }^{12}$ The inhibition of edema was recorded on a plethysmometer (UGO BASILE make) and expressed as \% inhibition. The results are given in Table 1 . Compounds $4 a-c$ showed $32-$ $34 \%$ inhibitiion in rats while aspirin and phenyl butazone at the same dose (100 $\mathrm{mg} / \mathrm{kg}$, p.o) produced $17 \%$ and $39 \%$ inhibition of $1 \%$ carrageenan-induced inflammation, respectively. The per cent protection for each compound was calculated using the following formula:

No. of wriths in test

$\%$ protection $=100-$

No of wriths in control $\times 100$

All the new compounds (4a-c) exhibited significant antiinflammatory activity comparable with that of phenylbutazone. Especially chloro substituent 
showed highest activity than methoxy. However, they were found to possess weak analgesic action with reference to aspirin.

Table-1. Evaluation of analgesic and antiinflammatory activities of compounds 4a-c

\begin{tabular}{cccc} 
Compound & \multicolumn{2}{c}{$\begin{array}{c}\text { Analgesic action } \\
\text { (\% protection of pain) }\end{array}$} & $\begin{array}{c}\text { Antiinflammatory } \\
\text { action (\% inhibition) }\end{array}$ \\
\cline { 2 - 4 } & Tail clip & Writhing & (Rat paw edema) \\
\hline 4a & 13 & 11 & 32 \\
4b & 12 & 12 & 32 \\
4c & 14 & 16 & 34 \\
Aspirin (100 mg/kg) & 55 & 46 & 17 \\
Phenylbutazone & 30 & 26 & 39 \\
(100 mg/kg) & & & \\
The results were of two observations. Values of 20\% inhibition of significant \\
$(p>0.01$ ) greater were
\end{tabular}

\section{Experimental section}

Melting points were determined in open glass capillaries on a polmon melting point apparatus and are uncorrected. ${ }^{1} \mathrm{H}$ NMR spectra were recorded on a Gemini (200 MHz) spectrometers (chemical shifts are recorded in $\delta, \mathrm{ppm}$ ); internal standard was TMS and IR spectra were recorded in $\mathrm{KBr}$ on a Perkin-Elmer biospectrometer. Elemental analyses were carried out with a Carlo Erba model 1106 Elemental Analyzer.

Preparation of 2a-c - G̣eneral Procedure. A mixture of $1 \mathrm{a}(15 \mathrm{mmol})$, thiourea (5 mmol) and iodine (15 mmol) was refluxed for $48 \mathrm{hr}$ in abs. ethanol (50 $\mathrm{mL}$ ). At this point TLC showed only a slight change in the substrate. After prolonged refluxing ( 4 to 5 days until TLC showed the absence of the ketone) the resulting hydride was dissolved in hot water. The solution was filtered while hot and the clear filterate was neutralized with a strong solution of ammonia. The resulting precipitate was washed with water and crystallized from ethanol.

5,6-Dihydro-4H-benzo[3,4]cyclohepta[d][1,3]thiazol-2-amine (2a). Yield 60\%, pale yellow, m.p. $250^{\circ} \mathrm{C}$ (lit., ${ }^{7}$ m.p. $250^{\circ} \mathrm{C}$ ); IR (Kbr) : v $3380 \mathrm{~cm}^{-1}$; ${ }^{1} \mathrm{H}$ NMR $\left(\right.$ DMSO-d $\left._{6}\right): \delta$ 2.18-2.40 (m, 2H, 5-H), 2.61-2.85 (m, 4H, 4 \& 6-H), 5.35 (br.s, $2 \mathrm{H}$, $\mathrm{NH}_{2}, \mathrm{D}_{2} \mathrm{O}$ exchangeable) and 6.50-7.25 (m, $\left.4 \mathrm{H}, \mathrm{Ar}-\mathrm{H}\right)$. 


\section{8,9-Dimethoxy-5,6-dihydro-4H-benzo[3,4]cyclohepta[d][1,3]thiazol-2-amine}

(2b). Yield $63 \%$, pale yellow crystals, m.p. $>290^{\circ} \mathrm{C}$ (lit., ${ }^{7}$ m.p. $>290^{\circ} \mathrm{C}$ ); IR $(\mathrm{KBr})$ : u $3385 \mathrm{~cm}^{-1} ;{ }^{1} \mathrm{H}$ NMR (DMSO-d $\left.\mathrm{d}_{6}\right): \delta 2.15-2.30(\mathrm{~m}, 2 \mathrm{H}, 5-\mathrm{H}), 2.60-2.85\left(\mathrm{~m}, 4 \mathrm{H}_{1} 4\right.$ \& $6-\mathrm{H}$ ), 5.35 (br.s, $2 \mathrm{H}, \mathrm{NH}_{2}, \mathrm{D}_{2} \mathrm{O}$ exchangeable), 3.90 (s, 3H, OMe), 3.95 (s, 3H, $\mathrm{OMe}), 6.50(\mathrm{~s}, 1 \mathrm{H}, 7-\mathrm{H})$ and $7.25(\mathrm{~s}, 1 \mathrm{H}, 10-\mathrm{H})$.

\section{8-Chloro-5,6-dihydro-4H-benzo[3,4]cyclohepta[d][1,3]thiazol-2-amine}

Yield $65 \%$, pale yellow prisms, m.p. $>290^{\circ} \mathrm{C}$ (lit., ${ }^{7}$ m.p. $>290^{\circ} \mathrm{C}$ ); IR $(\mathrm{KBr}$ ) : u 3388 $\mathrm{cm}^{-1} ;{ }^{1} \mathrm{H}$ NMR (DMSO-d $\left.\mathrm{d}_{6}\right): \delta 2.20-2.41(\mathrm{~m}, 2 \mathrm{H}, 5-\mathrm{H}), 2.60-2.85(\mathrm{~m}, 4 \mathrm{H}, 4 \& 6-\mathrm{H})$, 5.33 (br.s, $2 \mathrm{H}, \mathrm{NH}_{2}, \mathrm{D}_{2} \mathrm{O}$ exchangeable) and 6.48-7.22 (m, 3H, Ar-H).

Preparation of 3a-c - General procedure. A mixture of $2 \mathrm{a}(12 \mathrm{mmol})$ and phenacyl bromide $(12 \mathrm{mmol})$ in $50 \mathrm{~mL}$ ethanol was allowed to stand at room temperature overnight. The crystals, which separated, were collected by filtration and washed with a small amount of ethanol.

\section{2-(2-Imino-1,4,5,6-tetrahydro-2H-benzo[7,8]cyclohepta[d][1,3]thiazoi-1-yl)-1-} phenyl-1-ethene-1-ol (3a). Yield 68\%, m.p. $281.2^{\circ} \mathrm{C}$; IR (KBr) : v 3390, 3360, 2910, $2850 \mathrm{~cm}^{-1} ;{ }^{1} \mathrm{H}$ NMR (DMSO-d $): \delta$ 2.15-2.30 (m, 2H, 5-H), 2.60-2.85 (m, $4 \mathrm{H}, 4 \& 6-\mathrm{H}), 7.14(\mathrm{~s}, 1 \mathrm{H},=\mathrm{CH}), 8.66(\mathrm{br}, \mathrm{s}, 1 \mathrm{H},=\mathrm{NH}), 8.64(\mathrm{~s}, 1 \mathrm{H}, \mathrm{OH})$ and $6.50-$ $7.44(\mathrm{~m}, 9 \mathrm{H}, \mathrm{Ar}-\mathrm{H})$; (Found : $\mathrm{C}, 72.15 ; \mathrm{H}, 6.00 ; \mathrm{N}, 8.00$. Calcd. for $\mathrm{C}_{21} \mathrm{H}_{21} \mathrm{~N}_{2} \mathrm{OS}$ : C, $72.17 ; H, 6.06 ; \mathrm{N}, 8.01 \%)$.

2-(2-Imino-8,9-dimethoxy-1,4,5,6-tetrahydro-2H-benzo[7,8]cyclohepta[d][1,3] thiazol-1-yl)-1-phenyl-1-ethene-1-ol (3b). Yield $67 \%$, m.p. $260^{\circ} \mathrm{C}$ (dec.); IR $(\mathrm{KBr}): \mathrm{u} 3385,2910,2860 \mathrm{~cm}^{-1} ;{ }^{1} \mathrm{H}$ NMR (DMSO-d $): \delta 2.18-2.33(\mathrm{~m}, 2 \mathrm{H}, 5-\mathrm{H})$, 2.60-2.88 (m, 4H, 4 \& 6. $\mathrm{H}), 7.18(\mathrm{~s}, 1 \mathrm{H},=\mathrm{CH}), 8.78(\mathrm{br}, \mathrm{s}, 1 \mathrm{H},=\mathrm{NH}), 8.76(\mathrm{~s}, 1 \mathrm{H}$, $\mathrm{OH}), 3.95(\mathrm{~s}, 3 \mathrm{H},-\mathrm{OMe}), 4.00(\mathrm{~s}, 3 \mathrm{H},-\mathrm{OMe}), 6.48(\mathrm{~s}, 1 \mathrm{H}, 7-\mathrm{H}), 7.23(\mathrm{~s}, 1 \mathrm{H}, 10-$ $\mathrm{H})$, 6.25-7.25 (m,5H, Ar-H). (Found: C, 67.51; $\mathrm{H}, 5.90 ; \mathrm{N}, 6.79$. Calcd. for $\left.\mathrm{C}_{23} \mathrm{H}_{24} \mathrm{~N}_{2} \mathrm{O}_{3} \mathrm{~S}: \mathrm{C}, 67.62 ; \mathrm{H}, 5.92 ; \mathrm{N}, 6.88 \%\right)$.

\section{2-(8-Chloro-2-imino-1,4,5,6-tetrahydro-2H-benzo[7,8]cyclohepta[d][1,3]}

thiazol-1-yl)-1-phenyl-1-ethene-1-ol (3c). Yield $67 \%$, m.p. $242^{\circ} \mathrm{C}$ (dec.); IR (KBr) : $v 3398,3355,3905,3855 \mathrm{~cm}^{-1} ;{ }^{1} \mathrm{H}$ NMR (DMSO-d $): \delta 2.17-2.23(\mathrm{~m}, 2 \mathrm{H}, 5-\mathrm{H})$, 2.60-2.86 (m, 4H, $4 \& 6-\mathrm{H}), 7.20(\mathrm{~s}, 1 \mathrm{H},=\mathrm{CH}), 8.70(\mathrm{br}, \mathrm{s}, 1 \mathrm{H},=\mathrm{NH}), 8.77(\mathrm{~s}, 1 \mathrm{H}$, $-\mathrm{OH}$ ) and 6.65-7.46 (m, 8H, Ar-H). (Found : $\mathrm{C}, 65.60 ; \mathrm{H}, 5.25 ; \mathrm{N}, 7.30$. Calcd for $\left.\mathrm{C}_{21} \mathrm{H}_{20} \mathrm{CIN}_{2} \mathrm{OS}: \mathrm{C}, 65.69 ; \mathrm{H}, 5.25 ; \mathrm{N}, 7.29 \%\right)$. 
Preparation of 4a-c - General Procedure. A suspension of $3 a(20 \mathrm{mmol})$ in 60 $\mathrm{mL}$ ethanol was heated under reflux for $6 \mathrm{hr}$ after cooling, the rystals which separated were collected by filtration.

10-Phenyl-6,7-dihydro-5H-benzo[6,7]cyclohepta[1,2,-d]imidazo[2,1-b]

$[1,3]$ thiazole (4a). Yield 92\%, colourless crystals; m.p. $238.2^{\circ} \mathrm{C}$; ${ }^{1} \mathrm{H}$ NMR (DMSO$\left.\mathrm{d}_{6}\right): \delta 2.18-2.37(\mathrm{~m}, 2 \mathrm{H}, 6-\mathrm{H}), 2.66-2.87(\mathrm{~m}, 4 \mathrm{H}, 5 \& 7-\mathrm{H})$. (Found : $\mathrm{C}, 75.90 ; \mathrm{H}_{\text {, }}$ $5.00 ; \mathrm{N}, 8.81$. Calcd. for $\mathrm{C}_{20} \mathrm{H}_{16} \mathrm{~N}_{2} \mathrm{~S}: \mathrm{C}, 75.91 ; \mathrm{H}, 5.10 ; \mathrm{N}, 8.85 \%$ ).

\section{2,3-Dimethoxy-10-phenyl-6,7-dihydro-5H-benzo[6,7]cyclohepta[1,2,-d]}

imidazo[2,1-b][1,3]thiazole (4b). Yield $90 \%$, buff white powder; m.p. $280^{\circ} \mathrm{C}$ (dec.); ${ }^{1} \mathrm{H}$ NMR (DMSO- $\left.\mathrm{d}_{6}\right): \delta 2.15-2.31(\mathrm{~m}, 2 \mathrm{H}, 6-\mathrm{H}), 2.61-2.85(\mathrm{~m}, 4 \mathrm{H}, 5 \& 7-\mathrm{H})$, $7.56(\mathrm{~s}, 1 \mathrm{H}, 11-\mathrm{H}), 3.99(\mathrm{~s}, 3 \mathrm{H},-\mathrm{OMe}), 4.10(\mathrm{~s}, 3 \mathrm{H},-\mathrm{OMe}), 6.49(\mathrm{~s}, 1 \mathrm{H}, 4-\mathrm{H}), 7.22$ (s, 1H, 1-H) and 6.25-7.40 (m, 5H, Ar-H). (Found: $\mathrm{C}, 68.10 ; \mathrm{H}, 5.71 ; \mathrm{N}, 7.96$ Calcd. for $\mathrm{C}_{20} \mathrm{H}_{20} \mathrm{~N}_{2} \mathrm{O}_{2} \mathrm{~S}: \mathrm{C}, 68.15 ; \mathrm{H}, 5.72 ; \mathrm{N}, 7.94 \%$ ).

3-Chloro-10-phenyl-6,7-dihydro-5H-benzo[6,7]cyclohepta[1,2,-d]

imidazo[2,1-b][1,3]thiazole (4c). Yield 93\%, pale yellow needles; m.p. $268^{\circ} \mathrm{C} ;{ }^{1} \mathrm{H}$ NMR (DMSO- $\left.\mathrm{d}_{6}\right): \delta$ 2.17-2.33 (m, 2H,6-H), 2.68-2.86 (m, 4H, 5 \& 7-H), $7.55(\mathrm{~s}$, $1 \mathrm{H}, 11-\mathrm{H}), 6.45-7.25(\mathrm{~m}, 8 \mathrm{H}, \mathrm{Ar}-\mathrm{H}) \cdot \cdot($ Found : $\mathrm{C}, 68.44 ; \mathrm{H}, 4.29 ; \mathrm{N}, 7.99$. Calcd. for $\left.\mathrm{C}_{20} \mathrm{H}_{15} \mathrm{CIN}{ }_{2} \mathrm{~S}: \mathrm{C}, 68.46 ; \mathrm{H}, 4.31 ; \mathrm{N}, 7.98 \%\right)$.

Acknowledgements

The authors are thankful to the Director, and the Head, Division of Organic Chemistry-II, IICT for providing facilities.

\section{References}

1. S.R. Ramdas, P.C. Chennaiah, N.S. Chandra Kumar, M.V. Krishna, P.S. Srinivasan, Sastry and S. Apparao, Heterocycles, 19, 861 (1982).

2. T.R. Bosin and E. Campaigne, Adv. Drug Res., 12, 191 (1977).

3. E. Campaigne, D.R. Knapp, E.S. Neiss \& T.R. Bosin, Adv. Drug Res., 5 (1970).

4. B.D. Tilak, Tetrahedron, 9, 76 (1960).

5. J. McLean, V. Peesapati and G.R. Proctor, J. Chem. Soc., Perkin Trans 1, 98 (1979).

6. N. Lingaiah and V. Peesapati, Org. Prep. Proced. Int., 24, 27 (1992). 
7. N. Lingaiah, V. Peesapati, Org. Prep. Proced. Int., 25, 5 (1993).

8. G.R. Proctor, J. Chem. Soc., 4274 (1964).

9. R.M. Dodson and L.C. King, J. Am. Chem. Soc., 67, 2242 (1945).

10. R.A. Turner, Analgesics in screening method in pharmacology, $1^{\text {st }}$ Edn (Acad. Press, New York), 100 (1965).

11. R. Koester, R. Anderson and E.S. De Deer, Acetic acid for analgesic screening, Fed. Proc., 18, 412 (1957).

12. C.A. Winter, E.A. Risley and G.W. Nuss, Proc. Soc. Exp. Biol. Med., 11, 544 (1962).

Received on May 10, 2001 